\title{
Sviluppare e valutare competenze argomentative in matematica: un percorso per la scuola elementare
}

\author{
Developing and assessing argumentative \\ competences in mathematics: \\ an educational path for primary school
}

\author{
Anna Maria Brunero` e Monica Panero \\ ${ }^{\star}$ Istituto Faà di Bruno - Torino, Italia \\ ${ }^{\circ}$ Dipartimento formazione e apprendimento, SUPSI - Locarno, Svizzera
}

Sunto / Le competenze argomentative stanno assumendo oggigiorno un ruolo sempre più cruciale in ambito educativo all'interno di una società in continuo mutamento, che richiede al singolo capacità critiche e comunicative sempre più sviluppate. Come è possibile far evolvere e valutare competenze così strutturate alla scuola elementare? Questo lavoro descrive un percorso sviluppato con una classe quinta di scuola elementare quasi completamente estranea alla pratica di argomentare per iscritto in matematica. Un aspetto centrale della metodologia implementata risiede nel fatto che i criteri validi per produrre e valutare un'argomentazione sono costruiti con gli allievi, in una serie di lezioni incentrate sul feedback, sulla valutazione tra pari e sull'autovalutazione.

In particolare, abbiamo sperimentato le potenzialità di un uso formativo di quesiti INVALSI, nati per una valutazione esterna, di sistema, qui reinterpretati come un'occasione per discutere e argomentare in classe, tracciando un possibile percorso per lo sviluppo delle competenze argomentative in matematica.

Parole chiave: argomentazione; feedback; prove INVALSI; criteri di valutazione; abilità metacognitive.

\begin{abstract}
Argumentative competences are nowadays assuming an increasingly crucial role in education within a society in continuous change, which requires the individual to develop increasingly critical and communicative skills. How is it possible to make evolve and to evaluate such structured competences in primary school? This paper presents an educational path developed with a fifth-grade class which was almost completely unrelated to the practice of arguing in writing in mathematics. A central aspect of the implemented methodology lies in the fact that the valid criteria for producing and evaluating an argument are constructed with the students, through a series of lessons focused on feedback, peer evaluation and self-assessment. In particular, we have tested the potentialities of a formative use of INVALSI items, born for an external assessment, reinterpreted here as an opportunity to discuss and argue in the classroom, describing a possible way for the development of argumentative competences in mathematics.
\end{abstract}

Keywords: argumentation; feedback; INVALSI test; evaluation criteria; metacognitive skills.

\section{1 intodaturione}

Nell'era delle cosiddette "Four Cs 21st century skills" (Critical thinking, Creativity, Collaboration, Communication: National Education Association, 2015), I'argomentazione sta assumendo un ruolo sempre più importante tra le competenze fondamentali nella formazione di uno studente affinché sviluppi capacità critiche e comunicative, vitali per riuscire nella scuola e nel mondo del lavoro. Nonostante sia messa chiaramente in luce nei principali riferimenti istituzionali, questa esigenza formativa non è ancora del tutto entrata a far parte delle pratiche d'aula più usuali. L'argo- 
mentazione in matematica, in particolare in forma scritta, rimane una pratica ancora poco diffusa alla scuola elementare in quanto può sembrare difficile da sviluppare e soprattutto da valutare. Diventa quindi essenziale sviluppare metodologie didattiche che consentano di lavorare sulla verbalizzazione, sulla spiegazione e sull'argomentazione con gli allievi fin dalla scuola dell'infanzia. Questo articolo si propone proprio di presentare, analizzare criticamente e disseminare un'esperienza realizzata con alunni frequentanti la classe quinta elementare che avevano lavorato poco, se non per nulla, sull'argomentazione in matematica, soprattutto in forma scritta.

\subsection{Le competenze argomentative nei riferimenti istituzionali}

Nei quadri di riferimento delle indagini a livello internazionale e nei diversi programmi scolastici nazionali che ne sono influenzati, lo sviluppo di competenze argomentative appare come un obiettivo di apprendimento trasversale e transdisciplinare da perseguire fin dai primi anni di scuola. Per quanto riguarda la matematica, il framework PISA (OECD, 2017) include le azioni di «riflettere su argomentazioni matematiche e spiegare e giustificare risultati matematici» (OECD, 2017, p. 69) nel modello della literacy matematica come parte del processo di utilizzare concetti matematici, fatti, procedure e ragionamenti. Anche il framework di matematica del TIMSS 2019 (Lindquist, Philpot, Mullis \& Cotter, 2017) include, nel dominio cognitivo del ragionamento, il processo di giustificazione inteso come «fornire argomentazioni matematiche per supportare una strategia o soluzione» (Lindquist et al., 2017, p. 24).

In accordo con queste linee guida, in Italia - Paese nel quale è stata svolta l'esperienza didattica riportata in questo articolo - le Indicazioni Nazionali (MIUR, 2012) sottolineano che la matematica «contribuisce a sviluppare la capacità di comunicare e discutere, di argomentare in modo corretto, di comprendere i punti di vista e le argomentazioni degli altri». Tra i traguardi che l'allievo deve raggiungere per uno sviluppo delle competenze al termine della scuola elementare (sezione Matematica, MIUR, 2012) in Italia, si legge:

- «Riesce a risolvere facili problemi in tutti gli ambiti di contenuto, mantenendo il controllo sia sul processo risolutivo, sia sui risultati. Descrive il procedimento seguito e riconosce strategie di soluzione diverse dalla propria».

- «Costruisce ragionamenti formulando ipotesi, sostenendo le proprie idee e confrontandosi con il punto di vista di altri»»1.

Tuttavia, argomentare non è solamente una competenza da sviluppare ma può rappresentare anche, come afferma Morselli (2014), il fondamento di un efficiente metodo didattico focalizzato sulla costruzione di significati matematici. Per fare ciò, I'insegnante deve essere capace di cogliere e sfruttare le occasioni per discutere con i propri allievi di come e perché una certa strategia matematica funzioni e al contempo di analizzare, spesso a caldo nell'immediato, le spiegazioni date dagli studenti, cercando di far emergere gli impliciti. Come si può dedurre dai traguardi per lo sviluppo delle competenze sopra riportati, un contesto fertile in cui l'insegnante può creare opportunità per argomentare è individuato nelle attività di risoluzione di problemi. L'argomentazione infatti è strettamente interconnessa con il problem solving (Di Martino, 2017): per avere accesso al ragionamento risolutivo seguito

1. Anche nel Piano di Studio per la scuola dell'obbligo ticinese (DECS, 2015) si possono individuare dei traguardi di competenza molto simili, relativi alla risoluzione di problemi e alla costruzione di ragionamenti, nonché l'intero processo cognitivo Comunicare e argomentare. 
da un allievo abbiamo bisogno di informazioni sulle strategie e sui processi che ha attivato (ossia abbiamo bisogno che l'allievo ci spieghi come ha ragionato) e sulle giustificazioni delle scelte fatte (ossia abbiamo bisogno che l'allievo ci spieghi perché ha ragionato in quel modo).

Questa stretta connessione fra le competenze di problem solving e di argomentazione è anche sostenuta nel quadro di riferimento delle prove standardizzate a livello nazionale italiano, somministrate dall'istituto INVALSI. Nel framework di matematica (INVALSI, 2017) si individuano tre dimensioni valutative (conoscere, risolvere problemi e argomentare) e si definiscono come strumentali sia le competenze di problem solving sia quelle argomentative, perché entrambe necessarie allo svolgimento di compiti più ampi e complessi.

Proprio la valutazione della dimensione dell'argomentazione viene presentata all'interno del quadro di riferimento delle prove di matematica come uno dei limiti della rilevazione standardizzata a livello nazionale, dal momento che essa viene valutata secondo la scelta dell'affermazione corretta e l'individuazione della sua giustificazione tra quelle proposte, tralasciando la valutazione della capacità di produrre e poi giustificare un'affermazione a partire da una data situazione (INVALSI, 2017).

\section{Quadro teorico}

In questo paragrafo presenteremo e articoleremo gli elementi teorici che hanno guidato le nostre scelte metodologiche.

\subsection{Spiegazione, giustificazione e argomentazione in matematica}

Nel paragrafo precedente, abbiamo utilizzato più volte i termini "argomentazione", "spiegazione" e "giustificazione". Nel linguaggio comune questi termini sono spesso utilizzati come sinonimi, ma nel nostro caso non lo sono. È opportuno chiarire il significato con cui verranno impiegati in questo articolo, basandoci sulle distinzioni che si possono ritrovare in letteratura.

Alcuni ricercatori (Cobb, Wood, Yackel \& McNeal, 1992; Yackel, 2001) propongono una distinzione tra spiegazione e giustificazione, a seconda delle diverse funzioni che esse svolgono. Si fornisce una spiegazione per chiarire aspetti del proprio ragionamento matematico che potrebbero non risultare evidenti agli altri. In casi specifici, tale spiegazione svolge la funzione di giustificazione se è fornita per reagire alle contestazioni di apparenti violazioni dell'attività normativa matematica. Una giustificazione matematica è quindi un caso particolare di spiegazione matematica che fa riferimento più direttamente a proprietà matematiche o criteri condivisi.

In uno studio esplorativo sulle spiegazioni fornite durante l'attività di risoluzione di problemi da parte di allievi dai 3 agli 8 anni, Levenson e Barkai (2013) hanno identificato diverse tipologie di funzioni di una spiegazione, tra cui appunto quella giustificativa. Nel complesso le funzioni identificate sono quattro, qui rinominate descrittiva, giustificativo-procedurale, giustificativo-concettuale ed esplorativo-generalizzante.

1. Funzione descrittiva: la spiegazione serve per descrivere il processo di pensiero del solutore o il modo in cui ha risolto il problema; risponde alla domanda: «Come hai risolto il problema?». 
2. Funzione giustificativo-procedurale: la spiegazione serve per giustificare la ragionevolezza e la plausibilità di una strategia o soluzione; risponde alla domanda: «Perché hai risolto il problema in questo modo?».

3. Funzione giustificativo-concettuale: la spiegazione giustifica la strategia utilizzata sulla base di proprietà e generalizzazioni matematiche; risponde ancora alla domanda: «Perché hai risolto il problema in questo modo?» ma facendo riferimento a dei fondamenti teorici.

4. Funzione esplorativo-generalizzante: la spiegazione è un passo verso nuove esplorazioni che conducono a generalizzazioni della strategia; più che rispondere a una domanda esterna, è un momento in cui il solutore si pone delle domande: passa dal risolvere al porsi problemi.

Le spiegazioni che svolgono la prima funzione, come sostengono Yackel (2001) e Krummheuer (2000), possono avere un format narrativo dal momento che sono strettamente connesse con il processo comunicativo di spiegare come si è proceduto, passaggio per passaggio, per risolvere il problema. Le spiegazioni che ricoprono la seconda o la terza funzione, ossia le giustificazioni, hanno come obiettivo spiegare perché ossia chiarire la razionalità di un'azione (Krummheuer, 2000) fondandosi o meno su proprietà matematiche. Le spiegazioni che ricoprono l'ultima funzione, infine, possono generare famiglie di situazioni problematiche a cui appartiene il problema risolto e innescare ulteriori esplorazioni in cui queste famiglie diventano nuovi oggetti di ragionamento (Nunokawa, 2010) e possono perciò costituire il primo passo verso una dimostrazione matematica.

Esposte le diverse possibili funzioni di una spiegazione matematica, ed evidenziate quelle che in particolare la rendono una giustificazione, passiamo a considerare il suo ruolo in un'argomentazione matematica. Consideriamo la spiegazione come elemento costitutivo di un'argomentazione: quest'ultima quindi, dei tre termini che stiamo discutendo in questo paragrafo, assume un'accezione più ampia. A sostegno di questa affermazione, ci riferiamo al modello di Toulmin (1975) secondo cui un'argomentazione è un discorso che a partire da certi dati (data) porta a delle conclusioni (claim). A fare da ponte tra i dati e le conclusioni è la garanzia (warrant), ossia una serie di concatenazioni logiche dalle quali, partendo dai dati, derivano le conclusioni. Tale garanzia, a sua volta, è supportata da un fondamento (backing). Siamo consapevoli che per utilizzare tale modello come lente in campo educativo, esso deve essere adattato o integrato. In origine, infatti, tale modello si fonda sull'idea che la logica funzioni come la giurisprudenza (Toulmin, 1975), quindi come un processo in tribunale in cui l'avvocato deve difendere la propria tesi costruendo argomentazioni a favore, in modo predeterminato e statico. Diversi ricercatori in didattica della matematica hanno utilizzato questa lente teorica con successo nelle loro analisi, arricchendola e integrandola con altri quadri propri della ricerca in educazione (e.g., Arzarello \& Sabena, 2011; Durand-Guerrier, Boero, Douek, Epp \& Tanguay, 2011). Inoltre, come vedremo nel prossimo paragrafo, altri ricercatori come i già citati Krummheuer, Yackel e Cobb, hanno ampliato il modello di Toulmin, adattandolo al contesto educativo della classe in cui le argomentazioni vengono costruite e negoziate tra gli allievi e possono variare in itinere in modo dinamico.

In questo articolo, a seconda della sua funzione, identificheremo la garanzia come spiegazione o come giustificazione. A guidare il nostro lavoro vi è l'ipotesi che le quattro funzioni delle spiegazioni matematiche, presentate da Levenson e Barkai (2013) in ordine crescente di consapevolezza circa la risoluzione di un problema, possano suggerire un possibile percorso evolutivo da promuovere per far sviluppare le competenze argomentative degli alunni. 


\subsection{Dimensione sociale dell'argomentazione e norme socio-matematiche}

Lo studente, se viene costantemente stimolato a spiegare il proprio procedimento e le ragioni delle proprie scelte, ha l'opportunità di verbalizzare il proprio ragionamento e di beneficiare di questa attività individuale che lo aiuta a chiarire ed organizzare il proprio pensiero. Inoltre, una volta pronunciata, un'argomentazione non è più un prodotto interno, ma è socializzato e funge da atto comunicativo. Tenendo conto delle dinamiche di classe, Krummheuer (1995) osserva che un'argomentazione può anche essere costituita interattivamente da diversi partecipanti, composta da diverse proposizioni situate, non predeterminate ma negoziate dai partecipanti mentre interagiscono. Analogamente, ciò che conta come spiegazione accettabile in matematica può essere interattivamente costituito come una "norma socio-matematica" (Yackel \& Cobb, 1996) che viene elaborata dal gruppo classe come base condivisa per la comunicazione attraverso discussioni collettive guidate dall'insegnante. Per assicurare una buona comunicazione con l'insegnante e con i pari, lo studente deve essere guidato a formulare le proprie argomentazioni in modo corretto, chiaro e completo (nel seguito, ci riferiremo a questi tre criteri come "le 3C"). Un discorso argomentativo è:

- corretto, quando non ci sono errori di tipo matematico nella conclusione o nella spiegazione data;

- chiaro, se è comprensibile per un interlocutore (insegnante o compagni);

- completo, se tutti i passaggi del ragionamento che conduce dai dati alla conclusione sono esplicitati (Cusi, Morselli \& Sabena, 2017).

Altri progetti, tra cui ad esempio il progetto Avimes-Piemonte (De Luca, Demartini, Migliano, Savioli, Serratore \& Vio, 2008), a cui ci siamo ispirate per la nostra esperienza didattica, hanno lavorato esplicitamente con gli allievi di scuola elementare per identificare e condividere dei criteri espliciti per valutare argomentazioni matematiche. Affinché questi criteri siano interiorizzati dagli alunni e possano sostenere l'evoluzione delle loro spiegazioni, infatti, è importante che siano proprio gli studenti a individuarli, discuterli e costruirli interattivamente con l'insegnante, facendoli diventare così una norma socio-matematica della classe. È esattamente quello che ci siamo proposte di fare in questa esperienza didattica, mettendo in campo strategie tipiche della valutazione formativa, con I'obiettivo di fornire agli allievi degli strumenti per poter valutare il proprio lavoro e quello dei pari e per poter avanzare nella propria comprensione.

\section{Metodologia}

La metodologia e gli esempi che discuteremo in questo articolo si contestualizzano in un progetto più ampio che abbiamo condotto nel corso dell'anno scolastico 2017/18 in undici classi quinte di tre scuole primarie ${ }^{2}$ di Torino, in qualità di ricercatrici esterne, coinvolgendo circa 250 allievi e 11 insegnanti. L'obiettivo del progetto è stato quello di elaborare, sperimentare e analizzare possibili usi formativi di prove standardizzate, nello specifico le prove INVALSI di matematica, incentrando il lavoro 
su un feedback continuo, pensato per essere comprensibile all'allievo e utile per permettergli di progredire nello sviluppo delle sue competenze argomentative.

Con questa ipotesi di lavoro, alcuni quesiti delle prove di quinta, somministrate dal 2009 ad oggi, sono stati selezionati e riadattati allo scopo di raccogliere argomentazioni prodotte dagli allievi. A tal fine, abbiamo progettato con gli insegnanti di classe ogni intervento in aula decidendo il tema e le modalità di lavoro (lavoro di gruppo, a coppie, discussione collettiva ecc.). Successivamente, abbiamo preparato i materiali e costruito una scheda individuale, con la quale raccogliere le argomentazioni scritte dei bambini. Tale scheda individuale era costituita da tre quesiti INVALSI relativi al tema della lezione, generalmente proposti a risposta aperta e sempre accompagnati dalla richiesta «Spiega come hai fatto/ragionato» o «Spiega perché la tua risposta è corretta». Nel caso in cui il quesito fosse a scelta multipla, abbiamo sempre aggiunto «Giustifica la tua scelta». La formulazione della domanda può influenzare la risposta data. Così una richiesta come «Spiega come hai fatto/ragionato» stimola in risposta una spiegazione con funzione principalmente descrittiva; al contrario, «Spiega perché la tua risposta è corretta» o «Giustifica la tua scelta» spinge verso spiegazioni di tipo giustificativo.

Tali richieste sono state aggiunte ai quesiti INVALSI originali, che per diverse ragioni (tra cui vincoli di tempo e necessità di una valutazione oggettiva, vedi par. 1.1) richiedono raramente una spiegazione della risposta fornita. Ciò non impedisce però all'insegnante di sfruttare questa possibilità in classe, anzi il docente è incoraggiato a utilizzare domande o problemi interessanti come risorsa per avere accesso al ragionamento e alle strategie messe in atto dagli studenti e stimolare la discussione in classe. In quest'ottica, sono state condotte diverse ricerche in Italia per indagare e promuovere un uso didattico delle prove. Si vedano, ad esempio, la «Ricerca didattica sull'uso delle prove INVALSI (area matematica) del primo ciclo del S.N.V. nella didattica in classe $»^{3}$ o il già citato progetto Avimes-Piemonte (De Luca et al., 2008). Individuando nei quesiti un'occasione per lavorare sull'argomentazione, ci siamo focalizzate sia sul prodotto (analizzando la risposta) che sul processo (analizzando la spiegazione). In parallelo con il modello di Toulmin, la risposta è la conclusione, mentre la spiegazione è la garanzia che permette allo studente di giungere a quella conclusione partendo dai dati forniti (testo e dati del problema, contesto, relazioni tra i dati, ...). I feedback forniti agli allievi relativamente alle argomentazioni da loro prodotte si sono evoluti da una lezione alla successiva con una crescente attenzione al processo risolutivo, al ragionamento e all'autoregolamentazione, in linea con le implicazioni didattiche riportate nello studio di Hattie e Timperley (2007): I'efficacia con cui un feedback può avvicinare l'allievo agli obiettivi di apprendimento dipende in parte dal livello a cui esso viene dato (sul compito, ossia sul prodotto; sullo svolgimento del compito, ossia sul processo; per favorire l'autoregolamentazione nell'allievo; sull'individuo in quanto persona, relativo ad aspetti affettivi).

Durante l'anno scolastico 2017/18, abbiamo incontrato gli allievi il più regolarmente possibile, circa una volta ogni sei settimane. Ciascuna lezione in classe durava in media due ore e trenta minuti ed era strutturata nel seguente modo (si veda la Figura 1):

1. Fase del feedback: si iniziava con un feedback sulla lezione precedente che, a partire dalle argomentazioni scritte dagli allievi nelle schede individuali di

3. Maggiori informazioni al seguente link: https://www.dm.unipi.it/webnew/it/ricerca/ricerca-didattica-sul luso-delle-prove-invalsi-area-matematica-del-primo-ciclo-del-snv-nella-didattica-in-classe 
fine lezione, prevedeva un lavoro a coppie o di gruppo seguito da una discussione collettiva, come preciseremo nel prossimo paragrafo;

2. Fase dell'attività: seguiva un'attività laboratoriale sul tema matematico scelto con l'insegnante, spaziando su tutti i nuclei disciplinari in base alle conoscenze in corso di apprendimento;

3. Fase di produzione individuale: si chiudeva distribuendo la scheda individuale relativa al tema della lezione.

Al termine di ogni lezione, le schede individuali degli studenti venivano raccolte e analizzate con l'insegnante di classe al fine di utilizzare le spiegazioni stesse dei bambini come base per organizzare e condurre la fase del feedback della lezione successiva.

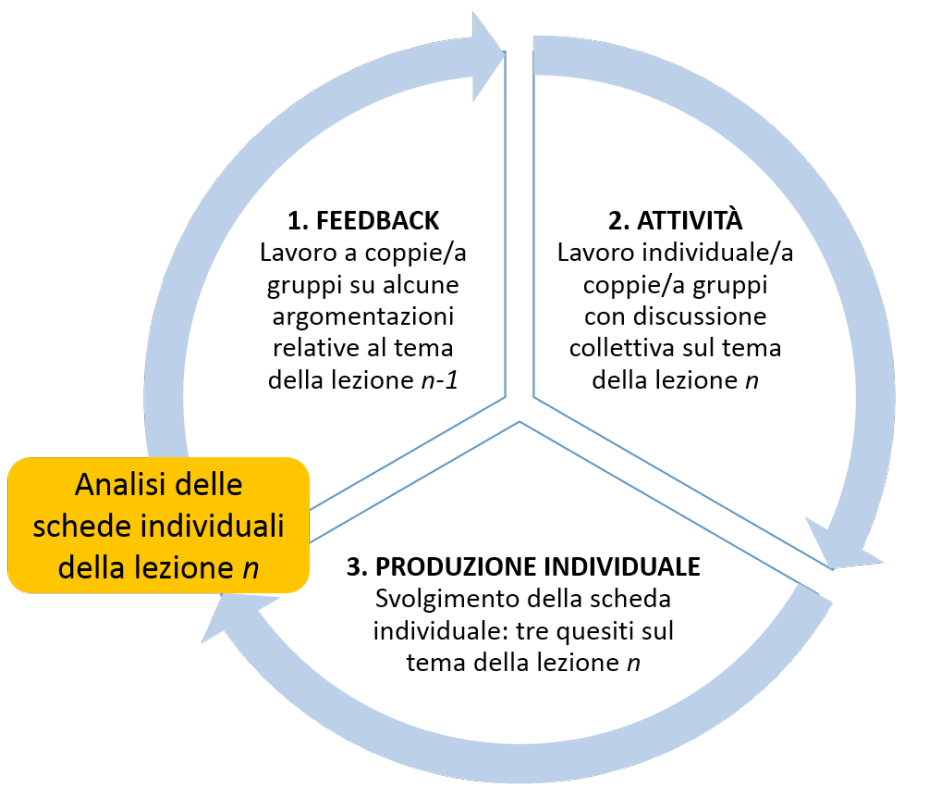

\section{Descrizione delle lezioni}

In questo paragrafo entreremo nel dettaglio delle varie lezioni svolte in una delle classi coinvolte nel progetto, riportando in particolare alcuni esempi di protocolli, estratti di discussione e reazioni degli studenti, legati principalmente alla fase del feedback. Prima di avviare il percorso, abbiamo analizzato con l'insegnante di classe i risultati dei suoi allievi ottenuti tre anni prima nella prova INVALSI di seconda elementare, in modo da individuare domande che allora erano risultate critiche per la maggior parte degli alunni: domande D2, D3, D5, D7, D9, D18 della prova L02 del 2015. Abbiamo quindi creato un breve test preliminare composto dai 6 quesiti individuati, resi a risposta aperta con richiesta di spiegazione (Allegato 0), e l'abbiamo riproposto agli allievi nel mese di ottobre 2017.

\subsection{Prima lezione: novembre 2017}

La prima lezione è stata dedicata all'ambito geometrico, in particolare al concetto di perimetro dei triangoli, tema che la classe stava ripassando in quel momento. 
Figura 2

Un esempio di grafico individuale (a sinistra) e collettivo (a destra).

\subsubsection{Fase del feedback - «Questa cosa di spiegare in matematica è una cosa nuova!»}

La prima lezione si è aperta con un feedback sul test preliminare relativo non solo alle risposte fornite, ma soprattutto alle spiegazioni scritte. Per affrontare questa analisi con gli alunni si è scelto di creare dei grafici, uno individuale per ciascun allievo e uno comune della classe, costituiti da due colonne suddivise in 6 caselle, ognuna relativa a una domanda del test: una colonna rossa riguardante la correttezza delle risposte e l'altra blu relativa alla validità della spiegazione (Figura 2).
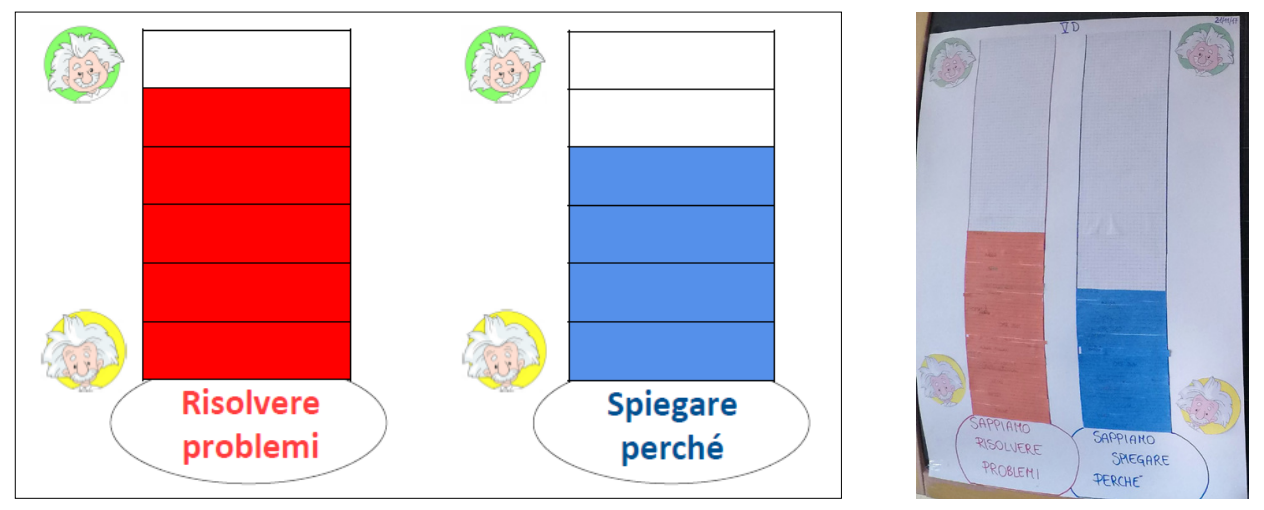

Utilizzando i termini del modello di Toulmin (1975), la colonna rossa del grafico si riferisce alla conclusione, mentre la colonna blu si riferisce alla garanzia che tale conclusione deriva dai dati forniti dal problema, integrati con opportune riflessioni e inferenze. I grafici utilizzati con i bambini hanno consentito di approcciare la valutazione di un'argomentazione matematica, nel caso di attività di risoluzione di problemi, nella sua interezza, ossia considerando nell'insieme tutte le componenti: dati, conclusioni e garanzia. La scelta di proporre la valutazione per mezzo di un grafico si fonda su uno strumento utilizzato nel progetto FaSMEd ${ }^{4}$ (Panero, 2017) risultato particolarmente significativo, perché ha permesso fin da subito di chiarire e condividere con gli allievi gli obiettivi del lavoro e rendere il singolo responsabile del proprio apprendimento. La fase del feedback si è svolta in modalità interattiva, con un'analisi dapprima individuale del grafico ricevuto. Come è possibile notare in Figura 2, oltre al grafico relativo al proprio andamento nel test preliminare, ogni alunno ha ricevuto i punti ottenuti (caselline colorate di rosso e di blu) da incollare all'interno del grafico della classe. Con la costruzione di un grafico collettivo abbiamo voluto coinvolgere tutti gli allievi nell'obiettivo di "far crescere le colonne della classe", soprattutto quella blu, sottolineando, con l'atto fisico dell'incollare anche un solo punto, che ciascuno di loro avrebbe potuto dare un contributo importante nel raggiungimento di questo obiettivo.

Durante questa prima lezione, il feedback si è principalmente focalizzato sugli aspetti affettivi e relativi alla visione che gli allievi avevano della matematica. Questa scelta è stata dettata dal fatto che gli alunni fossero perlopiù abituati ad argomentare solamente in forma orale e con modalità poco strutturate: per la maggior parte di loro

4. Formative Assessment in Science and Mathematics Education: https://microsites.ncl.ac.uk/fasmedtoolkit/ 
I'attività di argomentare per iscritto non era affatto familiare. La messa in evidenza delle due dimensioni (saper risolvere il problema e saper spiegare come/perché si risolve così) è risultata funzionale per esplicitare con gli alunni che tra di esse esiste un forte legame: è tanto importante saper risolvere un problema quanto saper spiegare ciò che si è fatto e perché si fa così.

Mentre il grafico della classe veniva costruito alla lavagna incollando i punti ottenuti dai singoli allievi, abbiamo condotto una discussione in plenaria, rilanciando le osservazioni degli allievi e proponendo continue domande stimolo, come per esempio «Quale colonna sarà più alta?», «Perché pensate che sarà più bassa quella blu?», «Sono collegate le due colonne?». Tali domande sono risultate efficaci per un'analisi del grafico, che si è poi focalizzata principalmente sulla colonna blu intitolata "Spiegare perché", che comportava un'abilità meno sviluppata per gli allievi.

Le capacità di autovalutazione rispetto ai propri limiti, risorse, possibilità e modalità di pensiero, relative alla capacità di utilizzare le informazioni apprese, ovvero imparare ad imparare (Commissione europea, 2007), sono emerse fin da subito con commenti come «Forse siamo stati più bravi a fare i problemi e non questa cosa di spiegare, perché è una cosa nuova e forse siamo meno esperti», «ll grafico si riempirà più di rosso che di blu». Questo strumento ha permesso, da un lato, di chiarire e condividere con i bambini tramite un linguaggio semplice e visivo gli obiettivi del nostro lavoro, dall'altro, di far emergere bisogni individuali e della classe rispetto a quegli obiettivi. Fin dall'inizio dell'esperienza, poi sempre di più lezione dopo lezione, gli allievi sono diventati consapevoli del fatto che spiegare per iscritto il proprio ragionamento o la propria motivazione di una scelta in matematica potesse risultare più difficile per loro rispetto a risolvere il problema, ma che fosse altrettanto importante e utile. Una delle domande stimolo che abbiamo posto di frequente è stata "A qualcuno è capitato, mentre scriveva la propria spiegazione, di avere un'idea su come risolvere il problema o di capire che il proprio ragionamento fosse sbagliato o incompleto?».

Questo tipo di feedback grafico, sia individuale che collettivo, è stato quindi mantenuto all'inizio di tutte le lezioni poiché fornisce un'immagine istantanea dell'andamento del singolo e della classe, commentabile e comprensibile agli allievi, senza alcun tipo di traduzione in voto o giudizio con finalità sommativa. Ci è capitato anche che alcuni alunni, talvolta, pur scrivendo molto, non siano riusciti ad ottenere neanche un punto in nessuna delle due dimensioni: questa eventualità non era stata considerata a priori e ha determinato una regolazione dell'uso dello strumento del grafico in itinere. Si è scelto, in questi casi, di non consegnare all'alunno il grafico bianco, in quanto sarebbe stato solo fonte di ansia, ma di dedicare un momento individuale parallelamente alla costruzione collettiva del grafico da parte dei compagni per ripercorrere la scheda con l'alunno in questione e chiarire, almeno oralmente, le sue spiegazioni. Sono stati gli alunni stessi a proporre, durante l'intervista, una correzione di alcune risposte o spiegazioni che avevano scritto, potendo così colorare i punti ottenuti sul loro grafico individuale e contribuire a quello collettivo, una volta rientrati in classe.

\subsubsection{Fase dell'attività e di produzione individuale}

Una volta conclusa la fase del feedback, abbiamo proposto un'attività intitolata "Cannucce e triangoli", ispirandoci al progetto m@t.abel. Abbiamo chiesto agli alunni, divisi in gruppi, di costruire dei triangoli piegando in due punti cannucce di lunghezza prestabilita $(18 \mathrm{~cm})$ per arrivare a mettere a fuoco le proprietà del triangolo, in particolare la disuguaglianza triangolare che lega le lunghezze dei tre lati. 
Affinché i bambini potessero dedurre questa proprietà a partire da un buon numero di diversi casi possibili, abbiamo distribuito cannucce graduate (con tacche poste a distanza di $1 \mathrm{~cm}$ l'una dall'altra) e un sacchetto contenente bigliettini numerati da 1 a 17. La consegna prevedeva di tirare a sorte la lunghezza dei primi due lati, garantendo così la casualità nella piegatura. Inoltre, ogni gruppo doveva annotare tutti i tentativi in una tabella: sia i casi riusciti sia quelli in cui non si formava un triangolo. Abbiamo riportato alla lavagna i risultati ottenuti dai vari gruppi, e attraverso la manipolazione del materiale e una discussione collettiva, i bambini sono arrivati a intuire che, nei casi in cui si forma il triangolo, la somma delle lunghezze di due lati è sempre maggiore della lunghezza del terzo lato.

Al termine della lezione, abbiamo distribuito la scheda individuale (Allegato 1), i cui quesiti sono un riadattamento degli item INVALSI: 1. D04 L05 20165, 2. D07 L06 2012, 3. D14 L05 2009.

\subsection{Seconda lezione: gennaio 2018}

La seconda lezione è stata dedicata a far emergere le $3 C$ come criteri per valutare un'argomentazione e a un'attività sulle proprietà delle operazioni e sulle espressioni.

\subsubsection{Fase del feedback - «Non si capisce! Abbiamo dovuto rileggerla mille volte!»} Per preparare la discussione, abbiamo preventivamente selezionato un quesito tra i più critici dal punto di vista dell'argomentazione (Figura 3) e, relativamente al quesito scelto, abbiamo selezionato quattro argomentazioni fornite dai bambini (Tabella 1) in modo che non contenessero errori nella risposta (conclusione), ma che differissero tra loro nella spiegazione (garanzia). Come criteri per la selezione abbiamo utilizzato le 3C: una spiegazione era corretta, chiara e completa, mentre alle altre tre mancava almeno uno di questi tre criteri.

3) Mario ritaglia quattro triangoli uguali e costruisce la girandola che vedi nella figura.

Figura 3

Terzo quesito della scheda individuale della prima

lezione (Allegato 1).

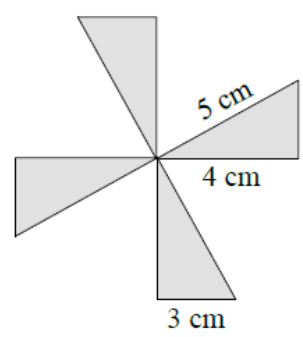

Scrivi un'espressione per trovare il perimetro della girandola:

Spiega perché la tua espressione permette di risolvere il problema:

5. Qui e in seguito, nel codice Dxx Lyy AAAA, xx indica il numero della domanda, yy indica il livello scolare e AAAA indica l'anno di somministrazione della prova INVALSI da cui è tratto il quesito selezionato. 
Nello specifico, la spiegazione A [«lo ho fatto questa espressione perché dovevi trovare la somma dei lati (cioè il perimetro) e moltiplicarla per 4 cioè i quattro triangoli rettangoli»] è chiara, corretta e completa; la spiegazione $B$ («Perché $i$ triangoli sono quatro ${ }^{6}$ o fatto per 4») è parzialmente corretta, ma non è chiara né completa (che cosa ha moltiplicato per 4?); la spiegazione C («perché ho moltiplicato i lati uguali per la misura dei lati»), oltre a non essere coerente con ciò che l'allieva ha scritto nella risposta, non è né corretta, né chiara, né completa (non spiega da dove ha preso il fattore moltiplicativo 4, ad esempio); la spiegazione D («Perché la mia espressione dice che dobbiamo moltiplicare le misure date per quattro che sono i triangoli») è corretta e chiara, ma non completa (non spiega che i prodotti parziali vanno poi sommati tra loro).

A

Scrivi un'espressione per trovare il perimetro della girandola:

$[(5+6+2)] x \cdot y=48$

Spiega perché la tua espressione permette di risolvere il problema: 10 HO RAITO QUUESTA ESPREzSIONE PERCHE DOVEII TROVARE LA SOMMA DEl LATI

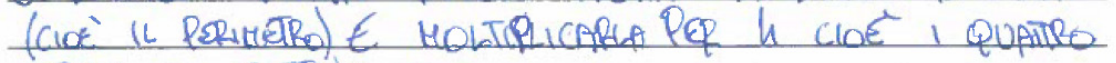
TRIANGOLI RETTANGOLI

$B^{7}$

Scrivi un'espressione per trovare il perimetro della girandola:

$3+4+5 \times 4=48$

Spiega perché la tua espressione permette di risolvere il problema:PER Cly I IRIAN(NOL) SONO QUATRO E D FATATO PER \&

C

Scrivi un'espressione per trovare il perimetro della girandola:

$(5 \times 4)+(4 \times 4)+(3 \times 4)=48$

Spiega perché la tua espressione permette di risolvere il problema:Cenche- ho moltiplisato i bt upuahi per la misura dei lativ

D

Scrivi un'espressione per trovare il perimetro della girandola:

$(5 \times 4)+(4 \times 4)+(3 \times 4)=$

Tabella 1

Quattro spiegazioni

selezionate per la discus-

sione a coppie e di classe.

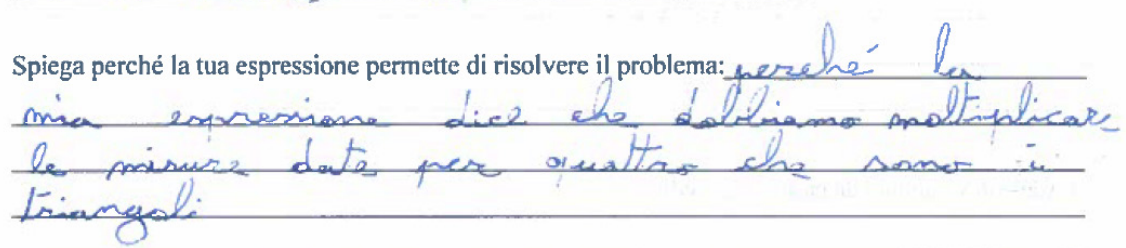

6. Gli errori di ortografia sono volutamente mantenuti nelle trascrizioni delle spiegazioni dei bambini. 7. Si noti che, in questa argomentazione, la conclusione non è corretta; mancano infatti le parentesi alla somma: $(3+4+5)$. Gli allievi, durante la discussione, hanno subito suggerito di inserirle; poi ci siamo concentrati sulle quattro spiegazioni. 
In classe, quindi, abbiamo consegnato una scheda contenente il problema scelto (Figura 3) e le quattro argomentazioni selezionate (Tabella 1); gli allievi hanno lavorato a coppie con la consegna di individuare "la migliore" tra tutte le spiegazioni, motivando la loro scelta. In questo modo, le produzioni di quattro allievi sono state messe a disposizione di tutti, adottando una delle strategie chiave di valutazione formativa: "attivare gli alunni come risorse educative gli uni per gli altri» (Black \& Wiliam, 2009 , p. 8). Nella discussione iniziale di queste prime lezioni volevamo che fossero gli alunni stessi a fornire un feedback per ogni argomentazione, individuando così le caratteristiche di una spiegazione che insegnante, allievi e compagni potessero ritenere valida in matematica. II feedback proposto si è focalizzato al livello del ragionamento/procedimento seguito per risolvere il compito, con una maggiore attenzione ai processi (Hattie \& Timperley, 2007). Nel complesso è stato possibile osservare nelle diverse classi una grande attenzione e precisione nel valutare le spiegazioni, con massima cura nei dettagli, più frequentemente di quanto previsto in fase di analisi a priori. II fatto di esprimersi su una spiegazione fornita da un loro compagno e non da un adulto (o da un insegnante) ha creato nella classe un clima di tranquillità. La terminologia dei tre criteri di correttezza, chiarezza e completezza è stata introdotta a partire dai commenti degli alunni. Motivando la scelta di una spiegazione piuttosto che un'altra, i bambini hanno esclamato:

- «Si capisce bene» O «Si capisce al volo»;

- «È la più dettagliata», «Spiega di più nei particolari, dice l'operazione che ha fatto» $\mathrm{O}$ «Le altre sono andate subito al punto mentre questa invece è più specifica»;

- «È tutto giusto».

Al contrario, per escludere una spiegazione, nella loro ricerca della migliore, hanno affermato:

- «Abbiamo dovuto rileggerla mille volte!» o «Non si capisce»;

- «Non c'è l'operazione, I'ha fatta nella sua mente» o «Manca un pezzo»;

- «L'operazione è sbagliata» $\mathrm{O}$ «Ha scritto ... ma voleva dire ....».

Man mano che gli allievi formulavano questi "criteri" di scelta o di esclusione per una spiegazione, li abbiamo scritti alla lavagna mantenendo le parole dei bambini e raggruppandoli in tre categorie. Abbiamo poi chiesto ai bambini di esprimere con una sola parola o un solo aggettivo tutte le spiegazioni che presentavano le caratteristiche di una stessa categoria. Abbiamo introdotto così le $3 \mathrm{C}$ che sono state richiamate ogni qual volta fosse richiesta una spiegazione, sia durante lo svolgimento delle schede individuali al termine di ogni lezione sia nelle discussioni collettive, nell'arco dell'intero progetto.

\subsubsection{Fase dell'attività e di produzione individuale}

Anche per questa attività, abbiamo ripreso una proposta del progetto m@t.abel, intitolata "Il bersaglio". Gli alunni, divisi in piccoli gruppi, dovevano trovare il numero maggiore che si potesse ottenere come risultato di un'espressione, utilizzando alcuni numeri assegnati, da considerare tutti e una volta sola, e potendo scegliere tra le quattro operazioni, anche ripetendole. II primo set di numeri assegnato era costituito da $2,3,5,10$. L'attività continuava con l'introduzione del numero 1 , elemento neutro della moltiplicazione, in un primo momento, e del numero 0 , elemento neutro dell'addizione e assorbente della moltiplicazione, in un secondo momento. Infine, si è resa più sfidante la situazione introducendo il numero decimale 0,5 . Per mezzo 
della discussione guidata dall'insegnante sono stati condivisi e analizzati i risultati, le espressioni utilizzate e le strategie seguite da ogni gruppo e si è ragionato sulla loro efficacia per ogni fase dell'attività, riprendendo nel complesso le proprietà delle quattro operazioni.

Al termine della lezione è stata distribuita la scheda individuale (Allegato 2) i cui quesiti sono ispirati ai tre item INVALSI: 1. D22 L06 2012, 2. D18 L05 2016, 3. D32 L05 2013.

\subsection{Terza lezione: marzo 2018}

La terza lezione è stata dedicata alla riformulazione di alcune spiegazioni usando le $3 \mathrm{C}$ e a un'attività sul tema delle frazioni, in particolare sul confronto e sull'equivalenza fra frazioni.

\section{4·3.1 Fase del feedback - «Manca quel pizzico in più»}

Per questa fase abbiamo ripreso le modalità di lavoro, risultate funzionali durante la seconda lezione: grafico individuale e della classe, lavoro a coppie su quattro argomentazioni relative a un quesito (il secondo dell'Allegato 2), seguito da una discussione collettiva guidata dall'insegnante. La consegna data alle coppie all'inizio della lezione è stata di provare ad assegnare i punteggi sia alla risposta al quesito sia alla spiegazione data, come se stessero costruendo i grafici individuali degli allievi in questione. Per quanto riguarda la risposta, i bambini potevano assegnare 0 punti se era quella errata e 1 punto se era quella corretta. Invece, per quanto concerne la spiegazione, abbiamo portato i bambini a riflettere sul fatto che accade spesso che non siano presenti tutti e tre i criteri di correttezza, chiarezza e completezza, senza però che la spiegazione sia comunque da rigettare totalmente. La presenza simultanea, per esempio, del criterio della correttezza e della chiarezza non implica necessariamente quello della completezza, per cui può risultare all'atto valutativo che una risposta possa essere corretta matematicamente, chiara per l'interlocutore, ma non completa nei vari passaggi. Per la spiegazione, quindi i bambini potevano assegnare 1 punto se erano presenti tutti i criteri, mezzo punto se ne mancava uno, 0 punti se mancavano due o tutti e tre i criteri. L'attenzione spostata interamente sul processo ha permesso agli alunni di rintracciare nelle varie spiegazioni la presenza o la mancanza dei tre criteri. II feedback si è spinto verso il livello di autoregolamentazione, per incoraggiare gli allievi a porsi le domande necessarie per valutare la presenza o meno dei criteri individuati, rileggendo un'argomentazione come se fosse la propria e provando a dare un "peso" (attraverso il punteggio) alla mancanza di uno o più criteri.

L'assegnazione dei punti in questa fase è stata fondamentale per poter interiorizzare ulteriormente i criteri di valutazione adottati per l'analisi delle spiegazioni proposte dagli alunni per iscritto e far riflettere la classe sulla necessità di introdurre il mezzo punto. Gli allievi hanno avviato spontaneamente, in alcuni casi, un processo di rielaborazione e riformulazione della spiegazione: «Se avesse scritto ... allora avrebbe preso un punto». Tale processo è stato incoraggiato e supportato nella discussione collettiva, in cui gli allievi hanno provato a completare, chiarire o correggere le spiegazioni selezionate, rendendo così operativi i criteri.

A questo proposito, risulta significativa la discussione sull'argomentazione riportata in Figura 4 ( «Sono necessari 7 camion. Perché 10 macchine stanno in un camion altre 10 in un altro e fanno 2 camion e si va avanti così, però un camion dovrà trasportare solo 2 macchine»). 
Figura 4

Una delle argomentazion discusse in aula relativa al secondo quesito dell'Allegato 2 .
2) II camion che vedi in figura può trasportare al massimo 10 automobili.

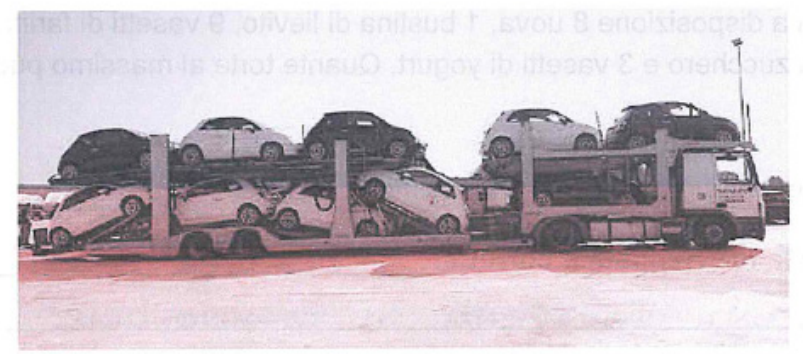

In fabbrica sono pronte 62 automobili da consegnare.

Qual è il numero minimo di camion, come quello in figura, necessario per consegnarle tutte?

Risposta: Sono necessari ..7.. camion.

Spiega perché la tua risposta è corretta: PERCHE 10 MACCHINE STANNO IN UN CAMION ALTRE 10 IN UN ALTRO E FANNO 2 CAMION E SI VA AUANZI COŠ, PERO UN CAMION DOVRÁ TRASPORTARE SOLO 2 MACCHINE.

Riportiamo qui di seguito un estratto della discussione (Ric. sta per "Ricercatrice").

1. Ric.: «Proviamo a dare i punti. Quanti punti ha preso questa spiegazione?».

2. G.: «Manca quel pizzico in più per arrivare a 0,5 [...] Potrebbero essere anche 70, 80, 90 macchine!».

3. S.: «Bastava, bastava mettere...».

4. Ric.: «Correggiamola, correggiamola! Bastava mettere?».

5. S.: «Bastava mettere i 6 ca... ehm... 6 per 10 ».

6. Ric.: " "E si va avanti così", poteva mettere tra parentesi $6 \times 10 »$.

7. I.: «No, io avrei messo "e si va avanti così per 6 volte"».

8. Ric.: «Eh! E poi ci scrivevi l'operazione, esatto».

9. M.: «Forse se avesse messo quello si poteva prendere uno 0,5 o addirittura un punto se lo faceva giusto, perché alla fine era corretta».

L'intervento di Giacomo ${ }^{8}$ (intervento 1) fa emergere non solo la comprensione del criterio valutativo, e cioè il fatto che la spiegazione non sia completa e non possa ottenere un punto, ma anche che gli servirebbero degli strumenti per poter definire ciò che manca, ossia per lui quel «pizzico in più». L'attenzione ai dettagli nella valutazione è molto forte da parte degli alunni, che cercano di riformulare l'argomentazione utilizzando il loro vocabolario e il loro lessico [si vedano i tentativi di Samuele e di lgor (interventi 3, 5 e 7)] in modo che soddisfi tutti i criteri per ricevere un punto pieno, come osserva Mattia (intervento 9).

8. Per la tutela della privacy dei bambini coinvolti, i nomi sono stati cambiati. 


\subsubsection{Fase dell'attività e di produzione individuale}

L'attività proposta durante la terza lezione era incentrata sul tema delle frazioni, richiamato attraverso la storia di cinque amici che decidono di partecipare a una gara di corsa. Allo scadere del tempo, nessuno di loro ha completato l'intero percorso. Ricevono comunque un attestato con su scritto la frazione di percorso completata: Marco ha percorso $\frac{1}{2}$ del percorso, Giada $\frac{1}{4}$, Valerio $\frac{8}{12}$, Giorgia $\frac{5}{6}$ e infine Luca $\frac{2}{3}$. Come si sono posizionati i cinque amici in classifica? Chi ha fatto più strada? Chi ne ha fatta di meno? Ogni allievo ha ricevuto la scheda relativa a un personaggio e ne ha rappresentato graficamente la parte di percorso completata su una striscia unitaria data (uguale per tutti), mobilitando così conoscenze matematiche relative alla frazione come parte-tutto e come operatore; per poter rispondere alle domande, però, ha dovuto cercare i compagni con gli altri quattro personaggi e operare un confronto dei percorsi lavorando in gruppo. Gradualmente, stimolati dalla guida dell'insegnante e dalla discussione con i pari, gli alunni hanno richiamato il significato di frazione equivalente, confrontando in particolare i percorsi di Valerio e di Luca. Alla lavagna queste ed altre frazioni sono state posizionate, o meglio appese, sulla linea dei numeri, ispirandosi a un'attività del percorso "Frazioni sul filo" (Robotti, Censi, Segor \& Peraillon, 2016).

Al termine dell'attività, abbiamo distribuito la scheda individuale (Allegato 3), i cui quesiti prendono spunto dai tre item INVALSI: 1. D30 L05 2016, 2. D19 L05 2014, 3. D11 L05 2016.

\subsection{Quarta lezione: aprile 2018}

Nell'ultima lezione abbiamo lavorato sull'autovalutazione e sulla valutazione tra pari, mentre l'attività proposta ha toccato il tema delle proprietà dei poligoni (triangoli e quadrilateri).

\subsubsection{Fase del feedback - «Quale sarà quella giusta?»}

Prima di consegnare i grafici individuali, abbiamo proposto che ogni alunno valutasse la propria scheda, avendo la possibilità di confrontarsi con i propri compagni a coppie o a gruppi di tre. Si è scelto di far confrontare allievi che avessero ottenuto globalmente punteggi simili, ma con risposte e spiegazioni anche molto differenti. Secondo Schwarz e Linchevski (2007), infatti, due allievi che hanno commesso errori simili difficilmente riusciranno ad autocorreggersi anche lavorando insieme, e allo stesso modo, un allievo che legge un metodo di risoluzione simile al suo, tendenzialmente parte dal presupposto che sia corretto.

I gruppi non erano quindi composti casualmente, bensì abbiamo fatto in modo di raggruppare allievi le cui schede fossero in un certo senso "complementari", come nell'esempio riportato in Tabella 2.

\begin{tabular}{|l|l|l|l|l|l|l|}
\hline \multicolumn{7}{|l|}{ Nostra valutazione della scheda individuale della terza lezione } \\
\hline GRUPPO 1 & $\begin{array}{l}\text { Quesito 1 } \\
\text { risposta }\end{array}$ & $\begin{array}{l}\text { Quesito 1 } \\
\text { spiegazione }\end{array}$ & $\begin{array}{l}\text { Quesito 2 } \\
\text { risposta }\end{array}$ & $\begin{array}{l}\text { Quesito 2 } \\
\text { spiegazione }\end{array}$ & $\begin{array}{l}\text { Quesito 3 } \\
\text { risposta }\end{array}$ & $\begin{array}{l}\text { Quesito 3 } \\
\text { spiegazione }\end{array}$ \\
\hline Allievo A & 1 & 1 & 1 & 0 & 0 & 0,5 \\
\hline Allievo B & 0 & 0,5 & 0 & 1 & 1 & 1 \\
\hline
\end{tabular}


Sui tre quesiti della scheda individuale relativa alla lezione precedente (Allegato 3), I'allievo $A$ ha risposto correttamente alle domande 1 e 2 e ha dato una spiegazione chiara, corretta e completa solo alla domanda 1 ; I'allievo B ha risposto correttamente alla domanda 3, ma ha dato una spiegazione valida alla domanda 2 (anche se la risposta è errata) e alla domanda 3. L'allievo A e l'allievo B hanno lavorato in coppia, confrontando le proprie schede, in modo che $A$ potesse aiutare $B$ nelle risposte $1 \mathrm{e}$ 2 e nella spiegazione 1. D'altra parte, $B$ avrebbe potuto aiutare A nella risposta 3 e nelle spiegazioni 2 e 3 . Non sapendo quale spiegazione fosse "la migliore", ciascun allievo è stato invitato a rileggere criticamente la propria e a difenderla agli occhi del compagno. Insieme dovevano decidere se entrambe o solo una meritasse un punto, oppure se entrambe dovevano essere riviste alla luce dei criteri, proponendo delle aggiunte o delle riformulazioni (usando una penna di un altro colore sulla propria scheda). Questo lavoro può funzionare nella misura in cui gli alunni sono spinti a ripensare e a verbalizzare il proprio ragionamento per spiegarlo al compagno e a fornire argomenti matematici convincenti per sostenere la propria risposta: la presenza delle spiegazioni scritte è, a questo scopo, un supporto importante. Questa ultima fase del feedback ha combinato una valutazione tra pari con un'autovalutazione, proponendo tutte e quattro le tipologie di feedback suggerite da Hattie e Timperley (2007): sul prodotto, sul processo, a livello auto-regolamentativo e affettivo.

Il grafico individuale è stato consegnato solo al termine di questo lavoro di gruppo e gli allievi hanno potuto confrontarlo con i punteggi auto-assegnati. II fatto di ricevere questa valutazione dell'insegnante solo in un secondo momento è stato vissuto da alcuni allievi con una lieve frustrazione. I gruppi più deboli sono stati aiutati dall'insegnante o dalle ricercatrici, ma gli altri gruppi hanno svolto il lavoro autonomamente, incontrando spesso il dubbio di quale fosse la spiegazione migliore tra due spiegazioni ugualmente corrette, chiare e complete, ma non identiche. Risulta interessante a questo proposito lo scambio avvenuto tra Marta ed Eva nella fase di auto-assegnazione dei punti alle spiegazioni da loro fornite al terzo quesito della scheda individuale (Figura 5 e Figura 6). 


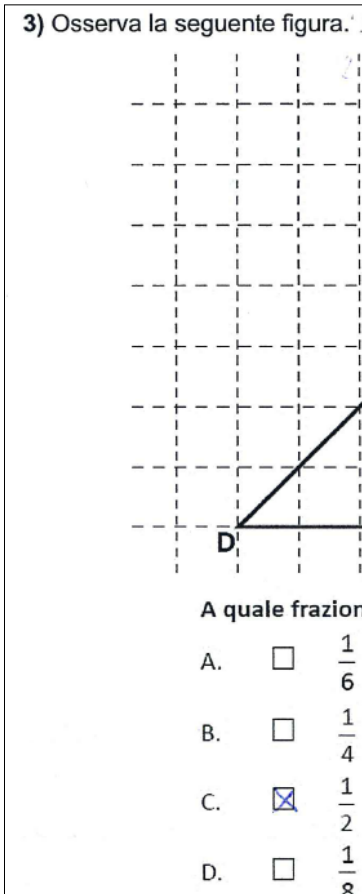

spiega perché: Perché contando l'area del trianodo DFE mi é venuto $36 \mathrm{~cm}^{2}$. Por ho contiondo l' area dof rettomedoi e mi é venuto $18 \mathrm{~cm}^{2}$. Alld fine ho lotto lo dilbrenzod ed imi é uscito 18 nerció lo brouzione sará 1

3) Osserva la seguente figura.

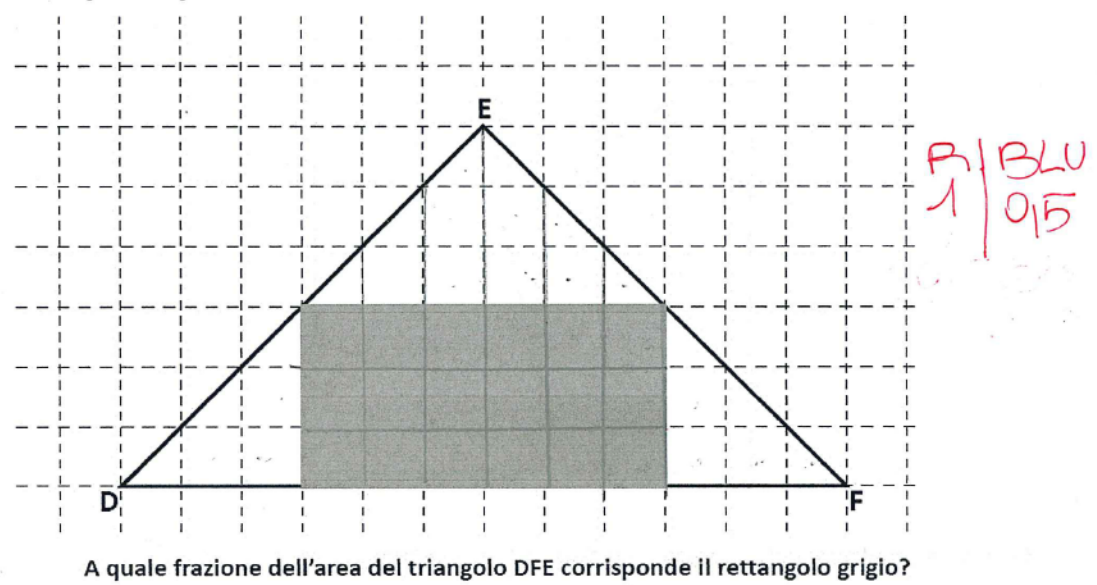
A. $\square \frac{1}{6}$
B. $\square \frac{1}{4}$
C. \&. $\frac{1}{2}$
D. $\square \frac{1}{8}$

spiega perché: Perchè se tu conti i ouvodrotimi olef rettansolo e poi conti tutti $i$ ownadratini del triensilo vedi quente volte si sta il rettangolo neltriangols 
Riportiamo qui di seguito un estratto della loro discussione.

1. M.: «Ok leggo io [legge la sua spiegazione, Figura 5]. Perché contando l'area del triangolo DFE mi è venuto $36 \mathrm{~cm}^{2}$ poi contando l'area del rettangolo mi è venuto $18 \mathrm{~cm}^{2}$ alla fine ho fatto la differenza e mi è uscito 18 , perciò la frazione sarà $\frac{1}{2}$ ».

2. E.: «No io non ho fatto così! [legge la sua spiegazione, Figura 6] Perché se tu conti i quadratini del rettangolo e poi conti tutti i quadratini del triangolo vedi quante volte ci sta il triangolo nel rettangolo».

3. M.: «Quindi?».

4. E.: «Quale sarà?».

5. M.: «Maestra, ma qual è quella giusta?».

6. Ric.: «Provate a deciderlo voi».

7. M.: "Secondo me la tua no».

8. E.: «Ma no guarda: 1, 2, 3, 4... [conta i quadratini del rettangolo] 36. Poi $1,2,3,4 \ldots$ [conta i quadratini del triangolo] ecco 18. Quindi ci starebbe due volte».

9. M.: «Perché se conti quanti quadrati ci sono nel rettangolo e poi tutti quelli del triangolo vedi quante volte ci sta il triangolo nel rettangolo... Mmm...».

10. E.: «Quindi la mia è la più giusta?».

11. M.: «Quanti punti ti daresti? 1 ० 0,5?».

12. E.: «lo 0,5 a me. A te darei tipo 1 ».

13. M.: «No 0,5 anche a me. Maestra, ma qual è quella giusta? Noi abbiamo deciso, ma non siamo tanto sicure».

Le richieste degli interventi 4 e 13 segnalano il bisogno delle due alunne di un confronto con la valutazione dell'insegnante per poter decidere con certezza la validità delle loro spiegazioni, tra loro differenti perché legate a due diverse strategie di risoluzione del problema.

Nel momento di confronto con il feedback fornito dal grafico individuale, abbiamo discusso soprattutto dei casi in cui i punteggi auto-assegnati dagli alunni si discostavano molto da quelli proposti dal loro grafico individuale.

\subsubsection{Fase dell'attività e di produzione individuale}

L'attività progettata per questa lezione si è basata sulla creazione di una versione "geometrica" del gioco classico dell'indovina chi. Gli alunni, suddivisi a coppie, hanno ricevuto il disegno di alcune figure geometriche con la richiesta di creare per ciascuna un identikit. La descrizione, che prevedeva il ripasso delle caratteristiche principali di ciascuna figura geometrica, nonché delle formule dell'area e del perimetro, è stata scritta su una carta a parte, mentre i disegni delle varie figure sono state ritirati dall'insegnante. A questo punto l'insegnante ha scambiato gli identikit fra le coppie ed è seguita una fase di matching: ogni coppia doveva riconoscere la figura corrispondente all'identikit ricevuto, trovarla tra i disegni disponibili, e incollarla sul retro della descrizione, completando cosi la realizzazione della carta da gioco. Poteva quindi iniziare il gioco vero e proprio in cui gli alunni, questa volta in gruppo, avevano a disposizione tutte le carte su un banco, girate dalla parte della figura, potendo consultare la descrizione sul retro in caso di bisogno; l'insegnante proponeva degli indizi per poter identificare una determinata figura e il gruppo, indizio dopo indizio, 
escludeva delle carte fino a trovare la figura in questione.

Al termine dell'attività, abbiamo distribuito la scheda individuale (Allegato 4), i cui quesiti sono una rivisitazione dei tre item INVALSI: 1. D04 L05 2016, 2. D16 L05 2012, 3. D6 L05 2015.

\section{Discussione dei risultati}

In questo paragrafo, categorizzeremo ed esemplificheremo le spiegazioni raccolte e analizzate in questa classe, in modo da riprendere le diverse funzioni di una spiegazione matematica proposte da Levenson e Barkai (2013). Mostreremo, inoltre, come I'uso formativo delle prove INVALSI qui proposto e le fasi del feedback implementate in questo percorso didattico abbiano permesso una graduale evoluzione delle argomentazioni scritte prodotte dagli allievi.

\subsection{Evoluzione delle spiegazioni: uno sguardo al singolo}

Durante la prima lezione, abbiamo raccolto parecchie spiegazioni non accettabili in matematica, ma è stato interessante analizzarne le tipologie, non essendo categorizzate in letteratura. Alcune le abbiamo classificate come affettive (ad esempio, «lo non sono sicuro infatti e l'ho fatto a caso»), altre come deleghe formali a un prototipo visto probabilmente sul libro di testo (ad esempio, "Perché I'ho già visto altre volte»), altre ancora come semplici ripetizioni della domanda (ad esempio, «Perché avevo trovato un'espressione»).

Come feedback per gli allievi che non sapevano bene da dove iniziare per scrivere una spiegazione matematica, abbiamo suggerito loro di provare a descrivere come si è risolto o si risolve il problema (la funzione descrittiva). Ci siamo quindi date come obiettivo quello di far sì che tutti gli alunni raggiungessero, nelle loro spiegazioni matematiche fornite per iscritto, almeno la funzione giustificativo-procedurale, che risponde alla domanda «Perché hai risolto così il problema?»; per gli allievi con una consapevolezza matematica maggiore, ci siamo prefissate che padroneggiassero anche quella giustificativo-concettuale, che risponde alla stessa domanda ma con espliciti riferimenti teorici. Nel perseguire questi obiettivi, è stato utile il lavoro sui criteri di chiarezza, completezza e correttezza. Nello specifico, per favorire l'evoluzione dalla funzione descrittiva a quella giustificativo-procedurale, cercavamo di innescare la domanda auto-regolamentativa «Perché ho fatto così?» al fine di completare e chiarire al meglio la spiegazione fornita; per favorire l'evoluzione dalla funzione giustificativo-procedurale a quella giustificativo-concettuale, tentavamo di far emergere la domanda auto-regolamentativa «Perché si può fare così in matematica?» al fine di completare ancora più nel dettaglio la spiegazione fornita, ponendo l'attenzione anche al linguaggio matematico utilizzato.

In questa classe abbiamo potuto identificare alcuni singoli casi di allievi le cui competenze argomentative sono migliorate molto rispetto all'inizio dell'esperienza. Presentiamo, di seguito, il caso di Alessandro e quello di Davide.

\subsubsection{Il caso di Alessandro}

Alessandro è un bambino molto timido e insicuro, con forti difficoltà in matematica, 
che nella prima lezione di novembre ha spiegato le proprie risposte sul piano affettivo ( «lo non sono sicuro infatti e I'ho fatto a caso») oppure ripetendo la domanda («Avevo trovato un'espressione»). Con Alessandro abbiamo lavorato anche individualmente, quando nessuna delle tre risposte da lui fornite aveva totalizzato punti per il grafico individuale e di classe. II fatto di fornirgli feedback individuali e di seguirlo più nello specifico nei laboratori proposti ha sortito effetti positivi. Alessandro ha acquisito maggior sicurezza nelle spiegazioni scritte, provando dapprima a realizzarne alcune di tipo descrittivo, come quella in Figura $7(«(2,50 \times 4)+(4,20 \times 3)=10+12,60=22,60$. Perché ho moltiplicato per quattro il costo del girasole perché ci sono 4 girassoli poi ho moltiplicato per tre il costo delle rose perché ci sono 3 rose e gli ho addizionati»); poi producendo spiegazioni giustificativo-procedurali corrette, chiare e complete, come quella in Figura 8 ( «Saverio. Perché ho diviso 1:4 che fa 0,25, 1:3 che fa 0,31 e 1:2 che 0,5 e quindi la somma minore è $\left.\frac{1}{4} »\right)$; infine cimentandosi con spiegazioni giustificativo-concettuali, come quella in Figura 9 («Perché sotto ha un angolo retto»).

Figura 7

Argomentazione con spiegazione descrittiva (quesito 3, Allegato 2 . gennaio 2018).
3) Dal fioraio sono indicati i seguenti prezzi per girasoli e rose:

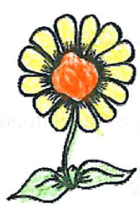

$€ 2,50$

Vuoi comprare questo mazzo di fiori ma non ha il cartellino.

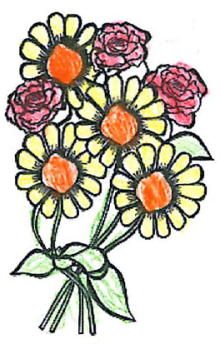

$€$ ?

Scrivi un'espressione per calcolare il prezzo del mazzo di fiori:

$(2,50 \times 4)+(4,20 \times 3)-10+12,60-22,60$

Spiega perché la tua espressione permette di risolvere il problema: PERCHE HO MOLTIPLICATO PER QUATTRO IL COSTO DEL, GRASOLE PERCHE CI SONO 4 GIRASSOLI POI HO MOLTIPULCATO PER TRE IL COSTO DELLE ROSE PERCHË CI SONO 3 ROSE E GLI HO AOOIZIONATI.

2) Saverio, Giorgio e Marco ricevono dai nonni la stessa somma di denaro.

Dopo una settimana a Saverio è rimasto $\frac{1}{4}$ dei soldi ricevuti, a Marco $\frac{1}{3}$ e a Giorgio $\frac{1}{2}$.

A chi sono rimasti meno soldi?

Risposta: SAVERIO

Spiega perché: PERCHE' HO DIVSSO 1:4 CHE FA 0.25 1:3 AHE FA 0,31E $1: 2$ CHE FA O,5 EQUNDI LA SOMMA MINORE E $\frac{1}{4}$ 
Figura 9

Argomentazione con spiegazione giustificativo-concettuale (quesito 1, Allegato 4 aprile 2018)

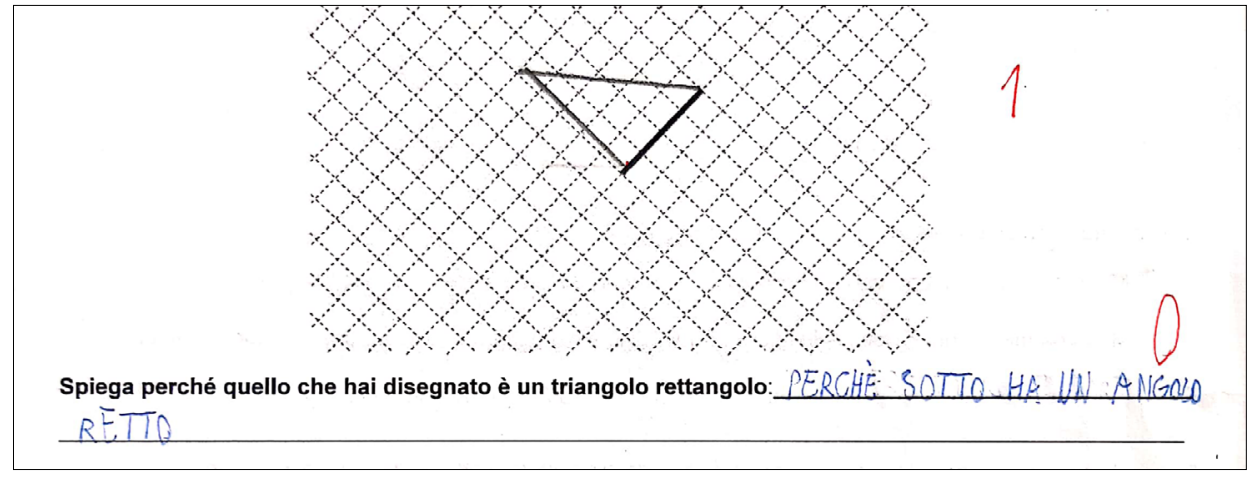

\subsubsection{Il caso di Davide}

Davide è un bambino sensibile e irrequieto, con spiccate capacità logiche e d'intuizione. Nella prima lezione, a novembre, aveva già fornito una spiegazione giustificativo-procedurale chiara, corretta e completa, relativa all'espressione per trovare il perimetro della girandola (Figura 3):

« $5 \times 4+3 \times 4+4 \times 4=48 \mathrm{~cm}$. lo ho scritto questa espressione perché: calcolando che ogni triangolo ha un lato da $3 \mathrm{~cm}$ uno da $5 \mathrm{~cm}$ e uno da $4 \mathrm{~cm}$, ho fatto $5 \times 4$ poi $3 \times 4$ ed in fine $4 \times 4$; poi ho sommato tutti i risultati per farmi venire come risultato il perimetro della girandola».

Nella seconda lezione, ha scritto spiegazioni giustificativo-procedurali che risultano solo parzialmente valide, come quella in Figura 10: "Ho fatto questa espressione perché ho calcolato i numeri delle marcherite e le ho moltiplicate per il numero del prezzo e la stessa cosa con le rose»; Davide doveva abituarsi a esplicitare tutti i passaggi per rendere complete le sue spiegazioni e a utilizzare un linguaggio più preciso per renderle più chiare. Nella scheda di marzo le sue spiegazioni giustificativo-procedurali sono risultate chiare, corrette e complete, come quella in Figura 11: «Ho risposto a Saverio perché $\frac{1}{4}$ è il numero più piccolo». Davide spiega perché ha risolto così il problema, ma non perché $\frac{1}{4}$ sia minore di $\frac{1}{3}$ e di $\frac{1}{2}$. Nell'ultima lezione Davide ha affinato le sue spiegazioni giustificativo-concettuali come quella in Figura 12: "Disegnando sulle figure e osservando mi sono reso conto che tutte le figure nel riquadro $A$, hanno $1 / 2$ assi di simmetria». Accompagnata dal disegno, questa spiegazione fa anche riferimento a che cosa significhi che una figura è simmetrica.

Scrivi un'espressione per calcolare il prezzo del mazzo di fiori:

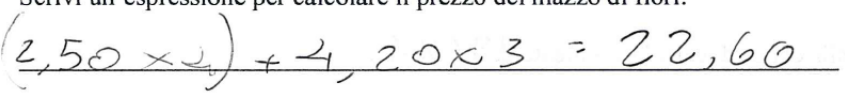

Spiega perché la tua espressione permette di risolvere il problema: Jo beto vucesthe

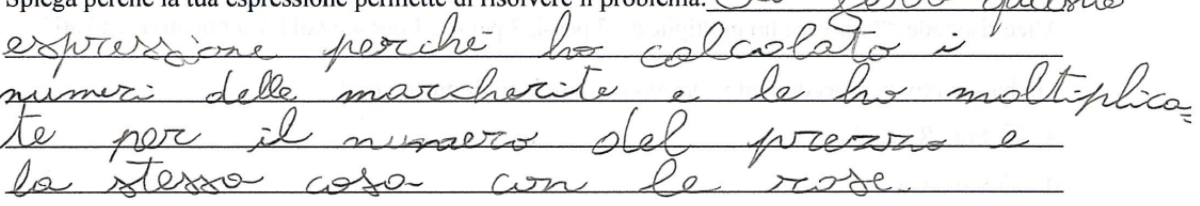

Figura 10

Argomentazione con spiegazione giustificativo-procedurale quesito 3, Allegato 2 gennaio 2018). 


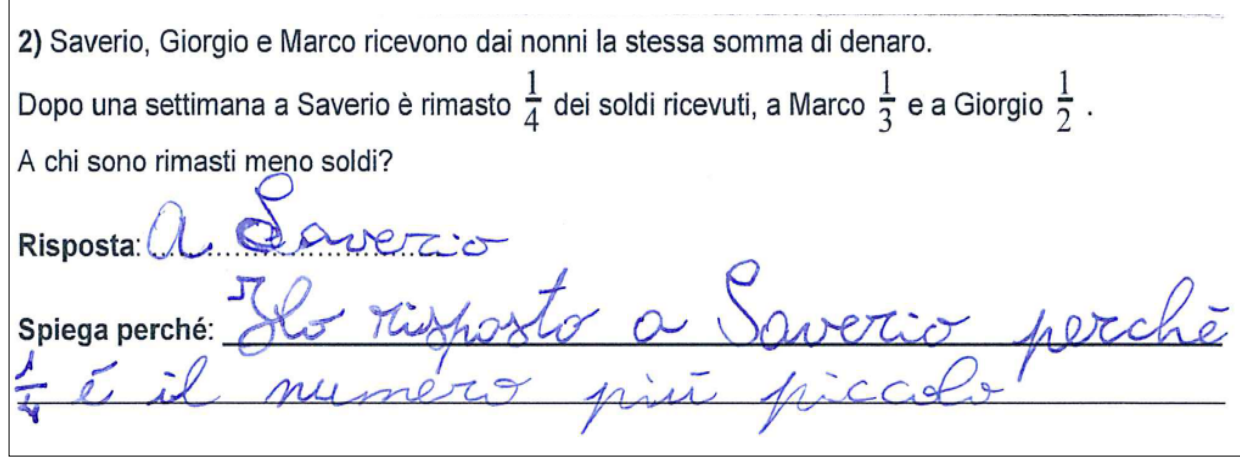

3) Mario ha suddiviso le seguenti figure in due gruppi utilizzando un criterio.

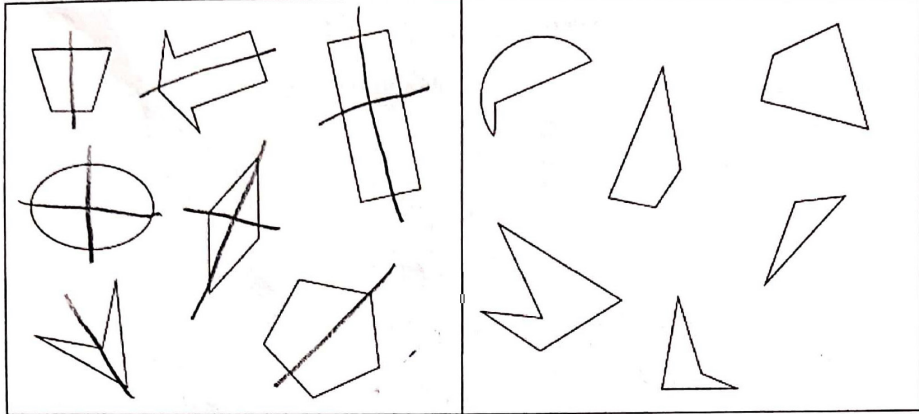

Gruppo A
Gruppo B

Quale criterio ha usato Mario per suddividere le figure?

A. $\square$ Mario ha messo nel gruppo A i poligoni e nel gruppo $B$ le figure che non sono poligoni.

B. Mario ha messo nel gruppo A le figure simmetriche e nel gruppo $B$ le figure che non sono simmetriche.

C. [] Mario ha messo nel gruppo A i quadrilateri e nel gruppo B le figure che non sono quadrilateri

Motiva la tua scelta (ricorda che puoi anche disegnare sulle figure e puoi andare per esclusione):

\subsection{Evoluzione delle spiegazioni: uno sguardo alla classe}

Possiamo inoltre identificare una globale evoluzione delle competenze argomentative degli allievi. Con ogni scheda individuale, abbiamo raccolto 57 spiegazioni (salvo casi di assenza di cui abbiamo tenuto conto), ossia 3 per ogni allievo, di cui riportiamo un'analisi in Tabella 3. Tra le spiegazioni con funzione descrittiva, abbiamo individuato alcune sotto-tipologie ricorrenti:

- Di tipo esperienziale, quando la spiegazione fa riferimento a un evento vissuto dall'allievo, a un fatto ricorrente nella sua vita quotidiana o scolastica, a sue osservazioni empiriche. In alcuni casi, ad esempio, gli alunni hanno fatto riferimento all'attività appena svolta.

- Per esclusione, quando la spiegazione descrive il procedimento di esclusione a una a una delle diverse opzioni di risposta fornite.

- Con verifica mediante esempio o schema, quando l'allievo sente la necessità di 
Tabella 3

Analisi delle funzioni

delle spiegazioni raccolte

durante l'esperienza

didattica. fare un esempio numerico o di abbozzare un disegno o uno schema per rendere più chiara la sua spiegazione.

Riteniamo questi casi interessanti dal punto di vista didattico per essere consapevoli, come insegnanti, delle possibili tipologie di argomentazione che i bambini possono produrre, soprattutto all'inizio di un percorso formativo sull'argomentazione nell'ambito del problem solving.

\begin{tabular}{|c|c|c|c|c|c|c|c|c|}
\hline Funzione spiegazione & 21.11 . & .2017 & 30.01 & .2018 & 16.03 & 2018 & 09.04 & 2018 \\
\hline $\begin{array}{r}\text { Descrittiva } \\
\text { esperienziale } \\
\text { per esclusione } \\
\text { verifica con esempio/schema } \\
\text { di cui matematicamente valide }\end{array}$ & 20 & $35 \%$ & $\begin{array}{rr}24 & \\
- \\
6 \\
- \\
8\end{array}$ & $53 \%$ & \begin{tabular}{ll|}
16 & \\
& 3 \\
& 1 \\
& 5 \\
& 3
\end{tabular} & $30 \%$ & \begin{tabular}{rr|}
17 & \\
2 \\
1 \\
7
\end{tabular} & $30 \%$ \\
\hline $\begin{array}{l}\text { Giustificativo-procedurale } \\
\text { di cui matematicamente valide }\end{array}$ & $\begin{array}{ll}7 & \\
& 1\end{array}$ & $12 \%$ & 8 & $18 \%$ & ${ }^{20}{ }^{11}$ & $37 \%$ & $\begin{array}{ll}3 & \\
& 1\end{array}$ & $5 \%$ \\
\hline $\begin{array}{l}\text { Giustificativo-concettuale } \\
\text { di cui matematicamente valide }\end{array}$ & \begin{tabular}{ll|}
13 & \\
& 6
\end{tabular} & $23 \%$ & - & - & 12 & $22 \%$ & ${ }^{23} 12$ & $40 \%$ \\
\hline $\begin{array}{r}\text { Diversa categorizzazione } \\
\text { (tutte non valide matematicamente) } \\
\text { affettivo } \\
\text { delega formale } \\
\text { ripetizione della domanda }\end{array}$ & \begin{tabular}{ll|}
14 & \\
3 \\
5 \\
6
\end{tabular} & $25 \%$ & $\begin{array}{l}- \\
- \\
2\end{array}$ & $4 \%$ & $\begin{array}{l}1 \\
2 \\
1\end{array}$ & $7 \%$ & $\begin{array}{r}- \\
1 \\
12\end{array}$ & $23 \%$ \\
\hline Vuote & 3 & $5 \%$ & 11 & $24 \%$ & 2 & $4 \%$ & 1 & $2 \%$ \\
\hline TOTALE & 57 & & 45 & & 54 & & 57 & \\
\hline
\end{tabular}

Analizzando la tabella, possiamo osservare che nelle prime due lezioni gli allievi hanno prodotto spiegazioni perlopiù descrittive, mentre quelle di tipo giustificativoprocedurale e giustificativo-concettuale sono aumentate nelle ultime due lezioni. In particolare, è anche cresciuta la percentuale di spiegazioni matematicamente valide in queste due categorie e risulta più evidente per le spiegazioni giustificativo-concettuali: nell'ultima lezione, metà delle giustificazioni fornite, con espliciti riferimenti a concetti teorici, sono risultate chiare, corrette e complete.

Il fatto che, per alcune lezioni, vi sia una percentuale più alta di un certo tipo di spiegazione può essere dovuto sia alla tematica e all'ambito affrontati, sia a come sono state poste le domande, in modo da incoraggiare maggiormente una certa funzione esplicativa. A questo scopo, per esempio, abbiamo volutamente riproposto due volte uno stesso quesito (n.1, Allegato 1 e Allegato 4), che non era stato discusso con i bambini tra una lezione e l'altra: la prima volta abbiamo chiesto agli allievi di spiegare come avessero proceduto, mentre la seconda volta abbiamo chiesto loro di spiegare perché il risultato fosse corretto; abbiamo effettivamente raccolto nel secondo caso spiegazioni quasi unicamente giustificativo-concettuali (16 casi su 19).

In un'altra occasione, abbiamo proposto un quesito di tipo cloze in cui erano dati quattro numeri razionali (due rappresentati mediante frazioni e due rappresentati mediante numeri decimali) ed era richiesto di posizionarli sulla retta numerica su cui erano già state identificate quattro possibili etichette (si veda il primo quesito dell'Allegato 3). Abbiamo appositamente chiesto agli allievi di spiegare come avessero ragionato per posizionare i numeri; volevamo infatti vedere se si sarebbero spinti oltre, cercando di giustificare la loro procedura. Ebbene, sono risultate più 
numerose le spiegazioni di tipo giustificativo-procedurale rispetto a quelle descrittive (attese, per il tipo di domanda).

\section{Conclusioni}

Le potenzialità della metodologia implementata si evincono dall'evoluzione delle argomentazioni degli alunni, di cui abbiamo presentato qualche esempio significativo. Questo mostra anche che le funzioni suggerite da Levenson e Barkai (2013) possono essere interpretate come vere e proprie tappe, attraverso le quali guidare l'alunno passo a passo.

A questo proposito il ruolo dell'insegnante diviene centrale nel suggerire vere e proprie domande stimolo e nel differenziarle in base alle tappe raggiunte dall'alunno; tali domande, se interiorizzate, lo direzionano nel suo percorso di consapevolezza, partendo da quesiti del tipo «Come hai risolto il problema?» per passare a «Perché hai risolto il problema in questo modo?» e a «Perché hai potuto risolvere il problema in questo modo in matematica?». Questo aspetto è fondamentale anche per quanto riguarda la valutazione delle argomentazioni fornite dagli alunni: chi valuta deve ben tener conto che le modalità e il lessico con cui richiede un'argomentazione veicola determinati aspetti a discapito magari di altri e può suscitare l'insorgere di un tipo di spiegazione invece di un altro. Consapevole di ciò, l'insegnante può favorire con le sue domande l'attivazione di un certo tipo di funzione, consentendo però sempre all'allievo di scegliere a sua discrezione la funzione che gli permette di esprimere in forma chiara, corretta e completa il suo pensiero.

Per accompagnare e stimolare l'evoluzione delle spiegazioni in termini di funzione, è stato fondamentale lavorare sui criteri per scrivere e riconoscere un'argomentazione valida in matematica, utilizzando diverse tipologie di feedback, con un focus crescente sul processo e sull'autoregolamentazione. Lezione dopo lezione, è stato possibile avviare un lavoro sul piano metacognitivo incoraggiando ogni allievo a imparare a porsi delle domande che lo potessero aiutare nello scrivere una spiegazione corretta ( «Ho ricontrollato le operazioni e i termini matematici che ho usato? Sono corretti?»), chiara («Ho riletto la mia spiegazione? Si capisce bene quello che volevo dire?») e completa («Ho messo tutti i passaggi?»). Come emerge dai risultati raccolti, questo percorso ha anche favorito lo sviluppo delle abilità metacognitive degli alunni nel corso dell'intero anno scolastico. L'abilità di riflettere sul proprio ragionamento in maniera consapevole sia sul processo di argomentazione e problem solving sia sulla valutazione è emerso durante le varie fasi del lavoro svolto. Gli alunni hanno avuto modo di riflettere sui processi attivati e hanno dimostrato di avere interiorizzato i criteri scelti per la valutazione delle argomentazioni a tal punto da estenderli alla loro pratica matematica anche al di fuori delle nostre lezioni, facendoli divenire veri e propri strumenti di autovalutazione. Lo si può evincere dall'intervento di un alunno che sostiene di aver imparato "qualcosa di nuovo» dal progetto "per esempio, per correggere pensare a, pensare alle $3 \mathrm{C}$ mi è servito molto, perché le $3 \mathrm{C}$ non le avevamo mai usate per correggere e possono aiutarci in matematica».

Il percorso messo in atto nei suoi aspetti metodologici ha delineato, inoltre, un possibile uso formativo delle prove INVALSI di matematica che, per tutti i risultati analizzati e discussi, si è rivelato un valido supporto, se riadattato per lavorare sull'argomen- 
tazione e se usato in dialogo con le conoscenze e le competenze matematiche in corso di apprendimento. In quest'ottica la dimensione sociale è stata determinante a diversi livelli per la riuscita del percorso, come è emerso durante l'ultima lezione, in cui un'alunna sostiene di aver imparato «qualcosa di nuovo» perché il progetto le ha insegnato «sì qualcosa di matematica e di geometria però ti insegna anche a confrontarti con i compagni per trovare la soluzione e a capire, perché se hai sbagliato con gli altri riesci a capire meglio il perché».

In un'esperienza di questo tipo, vi sono anche dei limiti. Innanzitutto, la scansione temporale degli interventi potrebbe essere resa più fitta e continuativa. Inoltre, per lavorare su competenze così complesse, quali quelle argomentative, il lavoro dovrebbe aver inizio fin dalla scuola dell'infanzia, a livello orale, e a partire dal primo ciclo della scuola elementare, anche a livello scritto. Le idee e gli scenari presentati e discussi in quest'articolo possono prendere vita molto prima con i piccoli allievi, nella speranza che lo sforzo di mettere nero su bianco in maniera chiara, corretta e completa il proprio ragionamento fornisca all'allievo anche un supporto per ragionare e apprendere in matematica, e non solo.

\section{Ringraziamenti}

Ringraziamo per la disponibilità e l'accoglienza la maestra Patrizia e i suoi allievi di quinta che hanno partecipato a questo progetto nella scuola primaria "Murialdo" di Torino.

\section{Bibliografia}

Arzarello, F., \& Sabena, C. (2011). Semiotic and theoretic control in argumentation and proof activities. Educational Studies in Mathematics, 77(2-3), 189-206.

Black, P., \& Wiliam, D. (2009). Developing the theory of formative assessment. Educational Assessment, Evaluation and Accountability, 21(1), 5-31.

Cobb, P., Wood, T., Yackel, E., \& McNeal, B. (1992). Characteristics of classroom mathematics traditions: An interactional analysis. American Educational Research Journal, 29, $573-$ 604.

Commissione europea. (2007). Competenze chiave per l'apprendimento permanente. Un quadro di riferimento europeo. Lussemburgo: Ufficio delle pubblicazioni ufficiali delle Comunità europee.

Cusi, A., Morselli, F., \& Sabena, C. (2017). Promuovere strategie di valutazione formativa in Matematica con le nuove tecnologie: I'esperienza del progetto FaSMEd. Annali online della didattica e della formazione docente, 9(14), 91-107.

De Luca, M., Demartini, L., Migliano, P., Savioli, K., Serratore, E., \& Vio, E. (Eds.). (2008). Argomentare: un "laboratorio" per le competenze. AVIMES-VALMAT.

DECS. (2015). Piano di studio della scuola dell'obbligo ticinese. Bellinzona.

Di Martino, P. (2017). Problem solving e argomentazione matematica. Didattica della matematica. Dalla ricerca alle pratiche d'aula, 1, 23-37. 
Durand-Guerrier, V., Boero, P., Douek, N., Epp, S. S., \& Tanguay, D. (2011). Argumentation and proof in the mathematics classroom. In Proof and proving in mathematics education (pp. 349-367). Springer, Dordrecht.

Hattie, J., \& Timperley, H. (2007). The power of feedback. Review of educational research, $77(1), 81-112$

INVALSI. (2017). Quadro di riferimento delle prove di matematica del sistema nazionale di valutazione. Disponibile in https://www.invalsi.it/invalsi/doc evidenza/2017/QdR2017 190 417.pdf (consultato il 26.08.2019).

Krummheuer, G. (1995). The ethnology of argumentation. In P. Cobb \& H. Bauersfeld (Eds.), The emergence of mathematical meaning: Interaction in classroom cultures (pp. 229269). Hillsdale, NJ: Erlbaum.

Krummheuer, G. (2000). Mathematics learning in narrative classroom cultures: studies of argumentation in primary mathematics education, For the Learning of Mathematics, 20(1), 22-32.

Levenson, E., \& Barkai, R. (2013). Exploring the functions of explanations in mathematical activities for children ages 3-8 year old: The case of the Israeli curriculum. In Proceedings of the 8th Congress of the European Society for Research in Mathematics Education (pp. 2158-2167).

Lindquist, M., Philpot, R., Mullis, I. V. S., \& Cotter, K. E. (2017). TIMSS 2019 Mathematics Framework. In I. V. S. Mullis \& M. O. Martin (Eds.), TIMSS 2019 Assessment Frameworks (pp. 11-25). Disponibile in http://timssandpirls.bc.edu/timss2019/frameworks/ (consultato il 26.08.2019).

MIUR. (2012). Indicazioni nazionali per il curricolo della scuola dell'infanzia e del primo ciclo di istruzione. Annali della Pubblica Istruzione, Numero Speciale. Le Monnier.

Morselli, F. (2014). Spiega perché, spiega come... Argomentare alla scuola dell'infanzia. In C. Sabena, G. Cerrato \& E. Scalenghe (A cura di), L'apprendimento nella scuola dell'infanzia. Riflessioni teoriche ed esperienze didattiche (pp. 127-142). Roma, Aracne.

National Education Association. (2015). An educator's guide to the "four Cs". Preparing 21st Century Students for a Global Society. Disponibile in http://www.nea.org/tools/52217. $\underline{\mathrm{htm}}$ (consultato il 26.08.2019).

Nunokawa, K. (2010). Proof, mathematical problem-solving, and explanation in mathematics teaching. In G. Hanna, H. N. Jahnke \& H. Pulte (Eds.), Explanation and Proof in Mathematics: Philosophical and Educational Perspectives (pp. 223-236). New York: Springer.

OECD. (2017), PISA 2015 Assessment and Analytical Framework: Science, Reading, Mathematic, Financial Literacy and Collaborative Problem Solving, revised edition, PISA, OECD Publishing, Paris. http://dx.doi.org/10.1787/9789264281820-en

Panero, M. (2017). Che cosa so fare con le frazioni?. Atti del VII Convegno Nazionale DI.FI. MA., 379-389.

Robotti, E., Censi, A., Segor, I., \& Peraillon, L. (2016). Frazioni sul filo. Strumenti e strategie per la scuola primaria. Collana Artefatti intelligenti. Erickson.

Schwarz, B. B., \& Linchevski, L. (2007). The role of task design and argumentation in cognitive development during peer interaction: The case of proportional reasoning. Learning and Instruction, 17, 510-531. 
Sviluppare e valutare competenze argomentative in matematica: un percorso per la scuola elementare /

Anna Maria Brunero e Monica Panero

Toulmin, S. (1975). Gli usi dell'argomentazione. (trad. it. by Bertoldi G.). Rosenberg \& Sellier.

Yackel, E. (2001). Explanation, justification and argumentation in mathematics classrooms. In M. Van den Heuvel-Panhuizen (Ed.), Proceedings of the 25th Conference of the International Group for the Psychology of Mathematics Education PME-25, vol. 1, (pp. 1-9). Utrecht.

Yackel, E., \& Cobb, P. (1996). Sociomathematical norms, argumentation, and autonomy in mathematics. Journal for Research in Mathematics Education, 22, 390-408.

\author{
Autori/Anna Maria Brunero ${ }^{\star}$ e Monica Panero ${ }^{\circ}$ \\ *Istituto Faà di Bruno - Torino, Italia \\ oDipartimento formazione e apprendimento - SUPSI, Locarno, Svizzera \\ brunero.annamaria@gmail.com, monica.panero@supsi.ch
}

